\title{
Balanced Folding Over a Polygon and Euler Numbers
}

\section{EL-Kholy $E^{*}$ and El-Sharkawey E}

Department of Mathematics, Faculty of Science, Tanta University, Tanta, Egypt

\begin{abstract}
In this paper we introduced a new folding over a polygon we called it balanced folding, then we proved that for a balanced folding of a simply connected surface $M$ there is a subgroup of the group of all homeomorphisms of $M$ that acts 1-transitively on the 2-cells of $M$. Also we explored the relationship between balanced folding and covering spaces. Finally we obtained a general relation of the Euler number of surfaces which may balance folded over a polygon and we also listed all the possibilities if $M$ is a sphere balanced folded over a triangle and we gave the subgroup mentioned above in each case.
\end{abstract}

Keywords: Surface; Cellular folding; Singularities; Cayley color graph; 1-Transitive; Universal covering; Euler number

\section{Introduction}

Let $K$ and $L$ be cellular complexes and $f:|K| \rightarrow|L|$ a continuous map. Then $f: K \rightarrow L$ is a cellular map if

(i) for each cell $\sigma \in K, f(\sigma)$ is a cell in $L$,

(ii) $\operatorname{dim}(f(\sigma)) \leq \operatorname{dim}(\sigma)$, [1]

A cellular map $f: K \rightarrow L$ is a cellular folding iff

(i) for each i-cell $\sigma^{i} \in K, f\left(\sigma^{i}\right)$ is an i-cell in $L$, i.e., $f$ maps $i$-cells to $i$-cells,

(ii) if $\bar{\sigma}$ contains $n$ vertices, then $\overline{f(\sigma)}$ must contains $n$ distinct vertices, [2].

A cellular folding $f: K \rightarrow L$ is neat if $L^{n}-L^{n-1}$ consists of a single $n$-cell, interior $L$. The set of all cellular folding of $K$ into $L$ is denoted by $C(K$, $L$ ) and the set of all neat foldings of $K$ into $L$ by $\mathrm{N}(K, L)$.

If $f \in C(K, L)$, then $x \in K$ is said to be a singularity of $f$ iff $f$ is not a local homeomorphism at $x$. The set of all singularities of $f$ corresponds to the "folds" of the map. This set associates a cell decomposition $C_{f}$ of $M$. If $M$ is a surface, then the edges and vertices of $C_{f}$ form a graph $\Gamma_{f}$ embedded in $M$ [3].

Now there is a graph $K_{f}$ associated to $C_{f}$ in a natural way. In fact the vertices of $K_{f}$ are just the $n$-cells of $C_{f}$ and its edges are the (n-1)-cells. If $e \in C_{f}$ is $(n-1)$-cell, then e lies in the frontiers of exactly two $n$-cells $\sigma$, $\sigma /$. We then say that $e$ is an edge in $K_{f}$ with end points $\sigma, \sigma /$. The graph $K_{f}$ can be realized as a graph $\tilde{K}_{f}$ embedded in $M$ as follows. For each $n$-cell $\sigma$, choose any point $\tilde{\sigma} \in \sigma$. If the $n$-cells $\sigma, \sigma /$ are end points of $e$, then we can join $\tilde{\sigma}$ to $\tilde{\sigma}^{\prime}$ by an $\operatorname{arc} \tilde{e}$ in $M$ that runs from $\tilde{\sigma}$ through $\sigma$ and $\sigma^{\prime}$ to $\tilde{\sigma}^{\prime}$, crossing $e$ transversely at a single point. Trivially, the correspondence $\sigma \rightarrow \tilde{\sigma}, e \rightarrow \tilde{e}$ is a graph isomorphism. Figure 1 illustrates this relationship in case $\mathrm{n}=2$.

It should be noted that the graph $K_{f}$ may have more than one edge joining a given pair of vertices. For instance, consider the cellular folding $f$ of the torus into itself with the cellular subdivision shown in Figure 2. The graph $K_{f}$ has just two vertices but two edges, see Figure 2.

\section{Balanced Folding}

Definition 1: Let $M$ be a compact connected surface, and $P_{n}$ a cell complex having $n$ 0-cells, $n$ 1-cells and just one 2-cell. Again a continuous map $f: M \rightarrow P_{n}$ is called neat folding if there is a cell decomposition $C_{f}$ of $M$ such that: (i) $f$ is a cellular map of $C_{f}$ onto $C\left(P_{n}\right)$.

(ii) for each closed cell $\bar{\sigma}$ of $C_{f}, f \mid \bar{\sigma}$ is a homeomorphism of $\bar{\sigma}$ onto a closed cell of $C\left(P_{n}\right)$.

To avoid trivial cases, we require that that each 0 -cell of $M$ is an end point of more than two 1-cells. Thus the 0-cells and 1-cells of this decomposition form a finite graph $\Gamma_{f}$ without loops (but possibly with multiple edges) and $f$ folds $M$ along the edges or 1-cells of $\Gamma_{f}[4]$.

Let $f: M \rightarrow P_{n}$ be a neat folding. Then for any $n$-cells $A$ and $B$ there is a homeomorphism $f_{A B}: A \rightarrow B$ given by $f_{A B}(a)=b$ iff $f(a)=f(b)$, where $a \in A$ and $b \in B$. We can always extend $f_{A B}$ to a homeomorphism, $\bar{f}_{A B}: \bar{A} \rightarrow \bar{B}$ , but there need not exist an extensions to any open neighbourhoods of $A$ and $B$. The following two examples explore this fact.

Example 1: Let $M$ be a desk in the plane $R^{2}$ with the cellular subdivisions shown in Figure 3. Let $P_{4}$ be a desk with four 0-cells, four 1-cells and one 2 -cell.

$$
\begin{aligned}
& \text { Define a map } f: M \rightarrow P_{4} \text { by } f\left(\sigma_{i}\right)=\sigma, i=1,2, \ldots, 9, \\
& f\left(e_{17}^{1}\right)=f\left(e_{3}^{1}\right)=f\left(e_{16}^{1}\right)=f\left(e_{2}^{1}\right)=f\left(e_{15}^{1}\right)=f\left(e_{1}^{1}\right)=\bar{e}_{1}^{1} \\
& f\left(e_{20}^{1}\right)=f\left(e_{13}^{1}\right)=f\left(e_{6}^{1}\right)=f\left(e_{18}^{1}\right)=f\left(e_{11}^{1}\right)=f\left(e_{4}^{1}\right)=\bar{e}_{2}^{1} \\
& f\left(e_{24}^{1}\right)=f\left(e_{23}^{1}\right)=f\left(e_{22}^{1}\right)=f\left(e_{10}^{1}\right)=f\left(e_{9}^{1}\right)=f\left(e_{8}^{1}\right)=\bar{e}_{3}^{1} \\
& f\left(e_{21}^{1}\right)=f\left(e_{14}^{1}\right)=f\left(e_{7}^{1}\right)=f\left(e_{19}^{1}\right)=f\left(e_{12}^{1}\right)=f\left(e_{5}^{1}\right)=\bar{e}_{4}^{1} \\
& f\left(e_{11}^{0}\right)=f\left(e_{3}^{0}\right)=f\left(e_{9}^{0}\right)=f\left(e_{1}^{0}\right)=\bar{e}_{1}^{0}, \\
& f\left(e_{12}^{0}\right)=f\left(e_{4}^{0}\right)=f\left(e_{10}^{0}\right)=f\left(e_{2}^{0}\right)=\bar{e}_{2}^{0} \\
& f\left(e_{15}^{0}\right)=f\left(e_{7}^{0}\right)=f\left(e_{13}^{0}\right)=f\left(e_{5}^{0}\right)=\bar{e}_{4}^{0} \text { and } \\
& f\left(e_{16}^{0}\right)=f\left(e_{8}^{0}\right)=f\left(e_{14}^{0}\right)=f\left(e_{6}^{0}\right)=\bar{e}_{3}^{0} .
\end{aligned}
$$

Let $A=\sigma_{4}, B=\sigma_{7}$ be the 2-cells shaded in Figure 3. Then there is a homeomorphism $f_{A B}: A \rightarrow B$ given by $f_{A B}(x, y)=f\left(x^{\prime}, y^{\prime}\right)$ iff $f(x, y)=f\left(x^{\prime}, y^{\prime}\right)$

*Corresponding author: EL-Kholy E, Department of Mathematics, Faculty of Science Tanta University, Tanta, Egypt, Tel: +20403344352; E-mail: entsar.elkholy@yahoo.com

Received March 23, 2016; Accepted April 06, 2016; Published April 12, 2016

Citation: EL-Kholy E, El-Sharkawey E (2016) Balanced Folding Over a Polygon and Euler Numbers. J Appl Computat Math 5: 296. doi:10.4172/2168-9679.1000296

Copyright: (c) 2016 EL-Kholy E, et al. This is an open-access article distributed under the terms of the Creative Commons Attribution License, which permits unrestricted use, distribution, and reproduction in any medium, provided the original author and source are credited. 


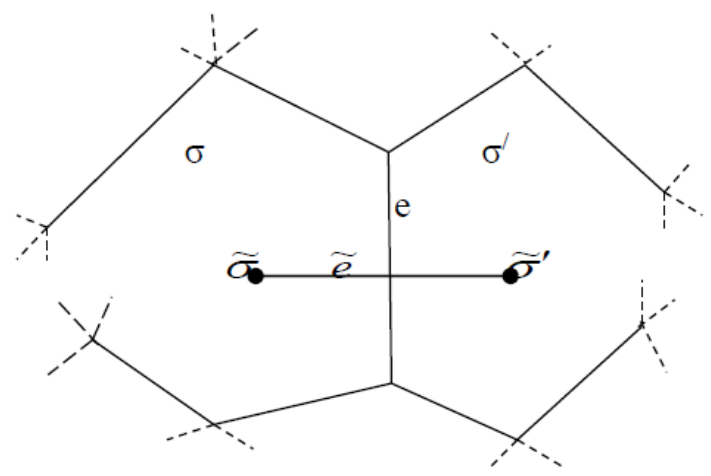

Figure 1: Relationship in case $n=2$.

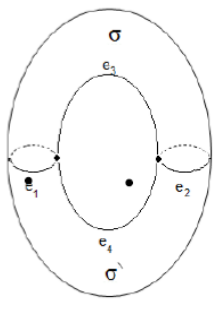

$\mathrm{T}$

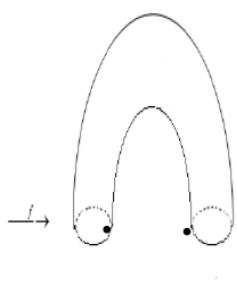

$f(\mathrm{~T})$

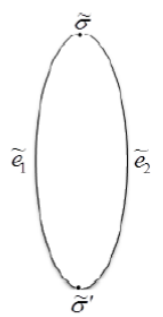

$\mathrm{K}_{f}$
Figure 2: Cellular subdivision.

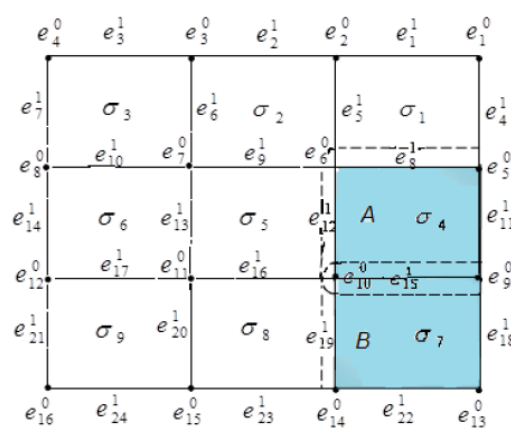

$M$

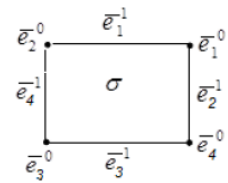

$P_{4}$
Figure 3: Cellular subdivisions 2.

where $(x, y) \in A$ and $(x, y) \in B$. This homeomorphism has an extension to a homeomorphism $\bar{f}_{A B}: \bar{A} \rightarrow \bar{B}$ given by $\bar{f}_{A B}(x, y)=\left(x^{\prime}, y^{\prime}\right)$ iff $f(x, y)=f\left(x^{\prime}, y^{\prime}\right)$, where $(x, y) \in \bar{A}$ and $\left(x^{\prime}, y^{\prime}\right) \in \bar{B}$. Now consider any open neighbourhoods $\tilde{A}, \tilde{B}$ of $\bar{A}, \bar{B}$ respectively. We see that there is no extension of $\bar{f}_{A B}$ to a homeomorphism $\tilde{f}_{A B}: \tilde{A} \rightarrow \tilde{B}$. This is because three 1-cells of $A$ are interior to $M$, while two 1-cells of $B$ have this property.

Example 2: Let $M$ be a sphere partitioned by the cells shown in Figure 4.

A cellular folding $f$ may be defined from $M$ to a polygon $P_{3}$. The vertices are labelled in such a way that vertices with the same image under $f$ are labelled alike.
Now, it can be checked that a homeomorphism $f_{A B}: A \rightarrow B$, (where $A$ and $B$ are the 2-cells shaded in Figure 4) cannot be extended to a homeomorphism of any neighborhoods $\tilde{A}, \tilde{B}$ of $\bar{A}, \bar{B}$ respectively. This is because the valencies of the vertices of the 2-cell $A$ are $12,4,4$ while those of $B$ are $12,8,4$.

Definition 2: We will call a neat folding: $f: M \rightarrow P_{n}$ a balanced folding if for all 2-cells $A, B$ and each homeomorphism $f_{A B}: A \rightarrow B$ given by $f_{A B}(a)=b$ iff $f(a)=f(b)$, we can extend $f_{A B}$ to a homeomorphism for any neighbourhoods $\tilde{A}, \tilde{B}$ of $\bar{A}, \bar{B}$ respectively.

We denote the set of all balanced foldings of $M$ into $P_{n}$ by $\boldsymbol{B}\left(M, P_{n}\right)$.

Example 3: Let $M$ be a sphere partitioned by the cells shown in Figure 5. The valencies of the vertices of each 2-cells are 4, 6 and 8 .

A neat folding $f$ may be defined from $M$ to a polygon $P_{3}$. The vertices are labeled in such a way that vertices with the same image under $f$ are labeled alike.

If we considered any 2-cells $A$ and $B$ of $M$ (e.g. the shaded 2- cells in Figure 5) then, it can be checked that a homeomorphism $f_{A B}: A \rightarrow B$, (where $A$ and $B$ are the 2-cells shaded in Figure 5) can be extended to a homeomorphism of any neighborhoods $\tilde{A}, \tilde{B}$ of $\bar{A}, \bar{B}$ respectively. This is because the vertices of the 2-cells $A$ and $B$ have the same valencies. It follows that $f$ is balanced.

\section{The Properties of the Graph $K_{f}$ of Neat Folding}

Let $\mathrm{f} \in \mathrm{N}\left(M, P_{n}\right)$, then $K_{f}$ has the following special features.

(a) Edge coloring: Let $e_{1}, e_{2}, \ldots, e_{n}$ be the 1-cells of $P_{n}$, we can regard the indices $i, i=1,2, \ldots, n$ "colors". Each edge of $K_{f}$ is mapped by $f$ to one

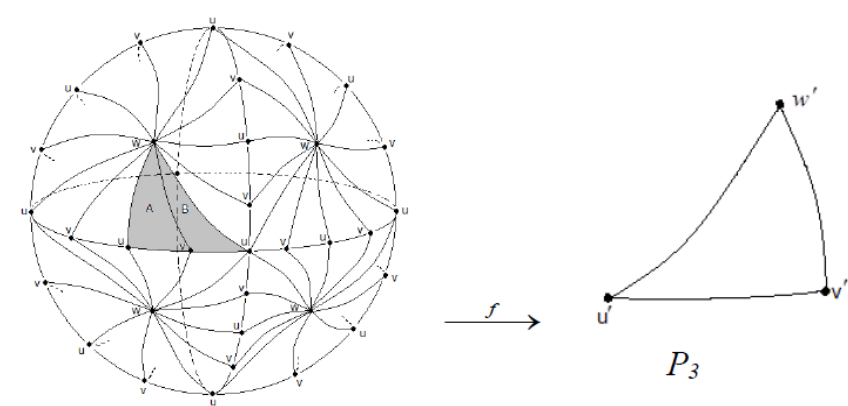

Figure 4: Sphere partitioned by the cells.

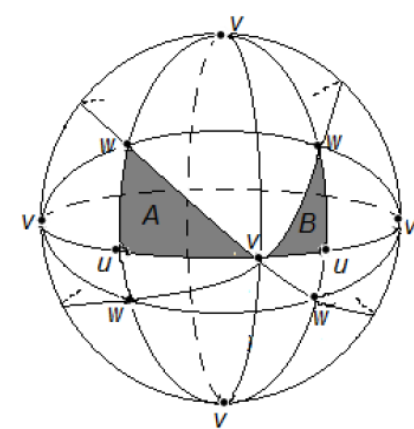

$M$

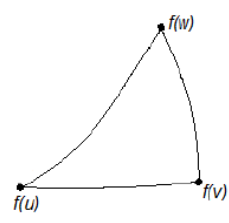

$P_{3}$
Figure 5: Sphere partitioned by the cells 2 . 
of these. We may then give $K_{f}$ an edge-coloring by assigning to each edge $e$ of $K_{f}$ the color $i$ of its image $f(e)=e_{i}$.

(b) Sources and sinks: If $A$ and $B$ are 2-cells of $M$ with a common 1 -cell in their frontiers, then $A$ and $B$ are given opposite orientations by $f$. It follows that each edge of the graph $K_{f}$ may be oriented in such a way that every vertex is either a source or a sink (where a vertex $u$ is a source if all the oriented edges with $u$ as a vertex begin at $u$, and is a sink if all the edges end at $w$ ), see Figure 6. For such a graph, every circuit has an even number of edges (and hence of vertices).

(c) Regularity: If $f \in \mathrm{N}\left(M, P_{n}\right)$, so that every 2-cell of $M$ is mapped homeomorphically by $f$ onto interior $P_{n}$, then the graph $K_{f}$ is regular. This follows immediately from the fact that the 1-cells in the frontier of each 2-cell is 1-1 correspondence under $f$ with those of $P_{n}$. It is also worth observing that every color $i$ occurs once in the set of colored edges at each vertex of $K_{f}$. Consequently, the valency of each vertex of $K_{f}$ is the cardinality of the set of 1-cells of $P_{n}$, that is to say, of the set of colors.

The properties of the graph $K_{f}$ we have already discussed suggest that in certain cases the graph $K_{f}$ may be a Cayley color graph. In the following we can show that this is indeed the case for a large class of balanced foldings.

Note first that, for any map $f: M \rightarrow N$, we can associate a group $G(f)$ namely the group of all homeomorphisms $h: M \rightarrow M$ such that $f o h=f$. In case $f \in \mathrm{N}\left(M, P_{n}\right)$, we may ask whether the induced action of $G(f)$ on the 2-cells of $M$ is transitive. In particular, we ask whether there is a subgroup $H(f)$ of $G(f)$ that acts 1-transitively on the set of 2-cells.

\section{The Action of the Group of Homeorphisms on the 2-Cells}

Theorem 1: Let $M$ be a simply connected surface, $f: M \rightarrow P_{n}$ be a balanced folding then there is a subgroup $H(f)$ of $G(f)$ that acts 1transitively on the set of 2-cells of $M$. Moreover $K_{f}$ is a Cayley color graph of the group $H(f)$.

Proof: Let $f \in \boldsymbol{B}\left(M, P_{n}\right)$. Let $A, B$ be 2-cells of the cell decomposition of $M$ associated by $f$. Then $f_{A B}: A \rightarrow B$ extends to a homeomorphism $\tilde{f}_{A B}: \tilde{A} \rightarrow \tilde{B}$, where $\tilde{A}$ and $\tilde{B}$ are open neighborhoods of $A$ and $B$ respectively.

Now let $C$ be a 2-cell such that $C \neq A$ and $C \cap \tilde{A} \neq \varphi$. Let $\tilde{f}_{A B}(C) \subset D$. Then there are open neighborhoods $\tilde{C}$ and $\tilde{D}$ of $C$ and $D$ such that $f_{C D}$ extends to a homeomorphism $\tilde{f}_{C D}: \tilde{C} \rightarrow \tilde{D}$, where $\tilde{f}_{C D}$ and $\tilde{f}_{A B}$ agree on $\tilde{A} \cap \tilde{C}$. Iterate this procedure to extend $f_{A B}$ to a map $F_{A B}: M \rightarrow M$.

The existence and uniqueness of this extension are guaranteed by the fact that $M$ is 1 -connected.

Now, to prove that $F_{A B}$ is onto, let $y \in M$ a non-singular point.

Then $y$ belongs to a 2-cell $\sigma$. Let $B_{1}, B_{2}, \ldots, B_{k+1}=\sigma$, be a sequence of 2 -cells such that $B_{i}, B_{i+1}$ are contiguous, $i=1,2, \ldots, k$. The sequence $B_{1}$,

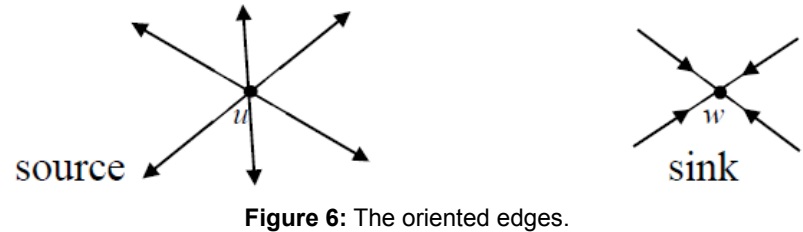

Figure 6: The oriented edges.
$B_{2}, \ldots, B_{k+1}$ of 2-cells is the image under $F_{A B}$ of a unique sequence $A_{1}$, $A_{2}, \ldots, A_{k+1}=\sigma^{\prime}$ of 2 -cells such that $A_{i}, A_{i+1}$ are contiguous, $i=1,2, \ldots, k$ and each $F_{A_{i} B_{i}}: A_{i} \rightarrow B_{i}$ extends to a homeomorphism $\tilde{F}_{A_{i} B_{i}}: \tilde{A}_{i} \rightarrow \tilde{B}_{i}$ where $\tilde{F}_{A_{i} B_{i}}$ and $\tilde{F}_{A_{i+1} B_{i+1}}$ agree on $\tilde{A}_{i} \cap \tilde{A}_{i+1}$. Hence $F_{A B}$ is onto.

We have now shown that $F_{A B}$ is a local homeomorphism of the simply connected manifold $M$ onto itself. In fact, $F_{A B}$ is a covering map. Thus $F_{A B}$ is a homeomorphism.

The set of all such homeomorphisms is the required group $H(f)$, which by its construction acts 1- transitively on the set of 2-cells.

The relationship of $H(f)$ to the graph $K_{f}$ is as follows:

Choose some 2-cells $A$. Thus $A$ is a vertex of $K_{f}$ Identify any other vertex (2-cell) $B$ of $K_{f}$ with the unique element $F_{A B}$ of $H(f)$ such that $F_{A B}(A)=B$.

It follows trivially that the graph $K_{f}$ is a Cayley color graph of $H(f)$, with generators $f_{B}=f_{A B}$, where $B$ runs through the set of 2-cells $B \neq A$ having a 1-cell in its common frontier with $A$.

Note that in general any neat folding $f$ of a surface $M$ to a surface $N$, the set of singularity forms the edges and vertices of a graph $\Gamma_{f}$. If $f$ is balanced, then the valencies of the vertices are invariant under any of the extended homeomorphisms $\tilde{F}_{A B}$. In particular, if $f \in \mathrm{N}\left(M, P_{n}\right)$ be such that $\Gamma_{f}$ is a regular graph embedded in $M$, then $f \in \beta\left(M, P_{n}\right)$. Moreover, if $M$ is simply connected, then $H(f)$ will acts 1-transitively on the set of 2-cells of $M$ and $K_{f}$ will be a Cayley color graph of the group $H(f)$.

Example 4: Let $M=S^{2}=\left\{x \in R^{3}:\|x\|=1\right\}$, be the unit sphere in the Euclidean 3-space. Let $f: M \rightarrow M$, be given by $f(x, y, z)=(|x|,|y|,|z|)$ . Then $f$ is a neat folding and the graph $\Gamma_{f}$ is a regular graph of valency 4 , with 6 vertices, twelve 1 -cells and eight 2 -cells. The image is the positive octant $P_{3}$ where $x \geq 0, y \geq 0, z \geq 0$ see Figure $7 \mathrm{a}$. Since $\Gamma_{f}$ is a regular graph, it follows that $f$ is a balanced folding and the graph, which is a Cayley color graph, has the form given in Figure $7 \mathrm{~b}$. Hence $H(f)$ is isomorphic to $Z_{2} \times Z_{2} \times Z_{2}$ and it acts 1-transitively on the set of eight 2-cells $A_{1}, A_{2}, \ldots$, $A_{8}$.

We now explore the relationship between balanced foldings and covering maps.

Theorem 2: Let $f \in \mathrm{N}\left(M, P_{n}\right)$, and let $p: \tilde{M} \rightarrow M$ be the universal covering. Suppose that

$\tilde{f}=f o p \in \beta\left(\tilde{M}, P_{n}\right)$ and that $G(p) \triangleleft H(\tilde{f})$. Then there is a subgroup $H(f)$ of $G(f)$, isomorphic to $H(\tilde{f}) / G(p)$, acting 1-transitively on the set of 2-cell of $M$ by $f$.

Proof: We first construct the group $H(f)$. Let $\tilde{h} \in H(\tilde{f})$, we now show that $\tilde{h}$ covers a (unique) homeomorphism $h: M \rightarrow M$, i.e. $h o p=p o \tilde{h}$. Let $a \in M$, and let $\tilde{a} \in p^{-1}(a)$. Put $b=p(\tilde{b})$, where $\tilde{b}=\tilde{h}(\tilde{a})$. The point $b$ is independent of the choice of $\tilde{a} \in p^{-1}(a)$. For if $p(\tilde{c})=a$, and $p(\tilde{d})=d$ where $\tilde{d}=\tilde{h}(\tilde{c})$, then there is an element $g \in G(p)$ such that $g(\tilde{a})=\tilde{c}$. Consider $g^{\prime}=\tilde{h}$ ogo $\tilde{h}^{-1}$. Then $g^{\prime}(\tilde{b})=\tilde{d}$. Since $G(p) \triangleleft H(\tilde{f}), g^{\prime} \in G(p)$. Thus $b=p(\tilde{b})=p(\tilde{d})=d$.

Now, define $h: M \rightarrow M$ by $h(a)=b$. Then $h$ is a homeomorphism of $M$, and, trivially, the set $H(f)=\{h: \tilde{h} \in H(\tilde{f})\}$ is a subgroup of $G(f)$ isomorphic to $H(\tilde{f}) / G(p)$. Thus there is an epimorphism $\theta: H(\tilde{f}) \rightarrow H(f)$ given by $\theta(\tilde{h})=h$.

Secondly, we show that $H(f)$ acts 1-transitively on the set of 2-cells of $M$ by $f$. Let $A, B$ be 2-cells of $M$ by $f$. Then there are 2-cells $\tilde{A}$ and $\tilde{B}$ of $\tilde{M}$ by $\tilde{f}$ such that $p(\tilde{A})=A$ and $p(\tilde{B})=B$. Let $\tilde{h}$ be the unique 


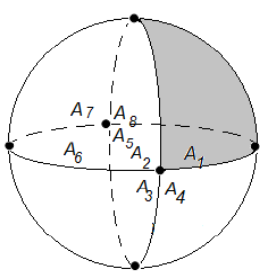

(a) $\mathrm{S}^{2}$ with the graph $\Gamma_{f}$

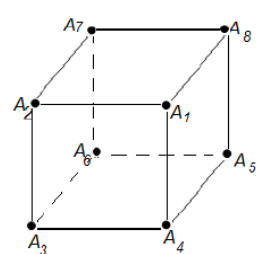

(b) The graph $K_{f}$ is a Cayley color graph
Figure 7: Cayley color graph.

element of $H(\tilde{f})$ such that $\tilde{h}(\tilde{A})=\tilde{B}$ and let $h=\theta(\tilde{h})$. Then $h(A)=B$, and there is only one such element of $H(f)$.

It should be noted that if $p: \tilde{M} \rightarrow M$ is a covering map, and $\tilde{f}=f o p$, where $f \in \mathrm{N}\left(M, P_{n}\right)$, then $\tilde{f} \in \beta\left(\tilde{M}, P_{n}\right)$ implies that $f \in \beta(M$, $\left.P_{n}\right)$.

Example 5: Let $M=P_{n}(R)$ be the real projective, n-space, and let $P_{n}$ be the n-polygon $\left\{t \in R^{n+1}: \sum_{i=1}^{n+1} t_{i}=1,0 \leq t_{i} \leq 1\right\}$. Define $f: M \rightarrow P_{n}$ by $f(\{x\})=\left(\left|x_{1}\right|, \ldots,\left|x_{n+1}\right|\right) /\|x\|$. Then $\breve{M}$ may be identified with $S^{n}$, and $p: \breve{M} \longrightarrow M$ is given by $p(x)=\{x\}$. In this case $G(p) \cong Z_{2}$ is generated by the map $g: S^{\mathrm{n}} \rightarrow S^{\mathrm{n}}, g(x)=-x$ and $H(\breve{f}) \cong\left(Z_{2}\right)^{n+1}$ is generated by the reflexions $g_{i}: R^{n+1} \rightarrow R^{n+1}, g_{i}\left(x_{1}, \ldots, x_{n+1}\right)=\left(x_{1}, \ldots, x_{i-1},-x_{i}, x_{i+1}, \ldots, x_{n+1}\right)$ and $\breve{f}(x)=(f o p)(x)=\left(\left|x_{1}\right|, \ldots,\left|x_{n+1}\right|\right) /\|x\|$ as above.

Theorem 3: Let $\tilde{f}$ and $f$ be as in Theorem 1 such that $G(p) \triangleleft H(\tilde{f})$. Let $\gamma$ : $L \rightarrow M$ be a regular covering. Then $H(g)$, where $g=f o \gamma$, acts 1-transitively on the set of 2-cells of $L$ by $g$.

Proof: Since $M$ is simply connected, for any other covering map $\gamma: L \rightarrow M$ there exists a universal covering map $h: \breve{M} \rightarrow L$ such that joh $=p$ (Figure 8).

Now $G(p) \cong \Pi_{1}(M)$ and $G(h) \cong \Pi_{1}(L)$. Since $\gamma: L \rightarrow M$ is regular $\gamma_{*} \Pi_{1}(L, y) \triangleleft \Pi_{1}(M, x)$, where $\gamma(y)=x$. There is isomorphism $\Phi: G(p) \rightarrow$ $\Pi_{1}(M)$ and $\Psi: G(h) \rightarrow \Pi_{1}(L)$ such that following diagram is commutative (Figure 9).

It follows from elementary group theory that, since $\Pi_{1}(L)$ is embedded in $\Pi_{1}(M)$ as a normal subgroup, then $G(h)$ is embedded by $\alpha$ in $G(p)$ as a normal subgroup. But $G(p) \triangleleft H(\tilde{f})$ by assumption. Hence $G(h) \triangleleft H(\tilde{f})$ and Theorem 2 can be applied for $g$, yielding that $G(g)=H(\tilde{f}) / G(h)$ acts 1-transitively on the set of 2-cells of $L$ by $g$.

\section{Euler Numbers of Balanced Folding onto a Polygon}

\section{General considerations}

Let $f \in \mathrm{N}(M, N)$, where $M$ and $N$ are surfaces. To avoid too many complications, let us suppose that $M$ is compact, connected and without boundary, and let $N$ be connected.

Since $M$ is compact the graph $\Gamma_{f}$ is a finite graph. Let $\Gamma_{f}$ divides $M$ into $k$ 2-cells, or faces, $A_{1}, A_{2}, \ldots, A_{k}$. In this case $f \mid A_{i}, i=1, \ldots, k$ is a homeomorphism onto the interior of $N$.

We can triangulate $N$ by a simplicial complex $T_{N}$ such that every vertex of the cell decomposition $C_{f}$ of $\partial N$ is a vertex of $T_{N}$. Let $T_{M}$ be the triangulation of $M$ induced by $f$.

Consider the faces $A_{1}, \ldots, A_{k}$ and their closures $B_{1}, \ldots, B_{k}$. Thus
$e\left(B_{i}\right)=e(N), i=1, \ldots, k$, where $e(X)$ is the Euler number of $X$. If we now calculate the Euler number $e(M)$ of $M$ using the triangulation $T_{N}$, then we can compare $e(M)$ with $\sum_{i=1}^{k} e\left(B_{i}\right)=k e(N)$. We note that for each vertex of $\Gamma_{f}$ with valency $v$ exactly $v$ vertices have been counted in the calculation of the Euler number $k e(N)$ of the disjoint union of $B_{1}, \ldots$, $B_{n}$. Likewise, every edge of $\Gamma_{f}$ appears twice in these calculations. Figure 10 which shows the neighborhood of a vertex with valency 4 , may help to clarify these remarks.

Thus to obtain $e(M)$ from $\sum_{i=1}^{k} e\left(B_{i}\right)$ we must subtract $(v-1)$ for each vertex of $\Gamma_{f}$ (of valency $v$ ) and add the number of edges of $\Gamma_{f}$. The first of these is $V$-nk, where $V$ is the number of vertices of $\Gamma_{f}$, and $n$ is the number of vertices of $\partial N$. The second is equal to $\frac{n k}{2}$. We conclude
that:

$$
e(M)=k e(N)+V-\frac{n k}{2}
$$

The case in which $N$ is the disc $D^{2}, e(N)=1$ and each 2-cell $A$ of $M$ is homeomorphic to $D^{2}$. Thus equation (1) now reduces to

$$
2 e(M)=k(2-n)+2 V
$$

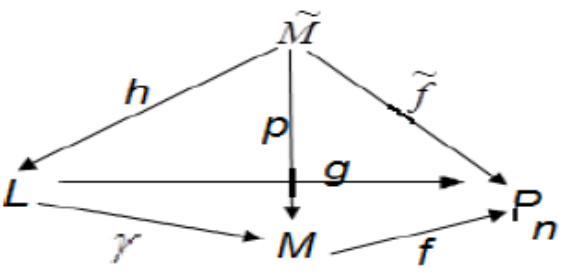

Figure 8: Universal covering map.

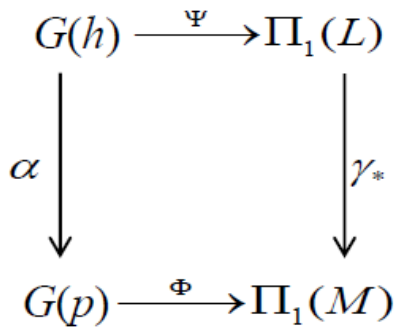

Figure 9: Universal covering map 2.

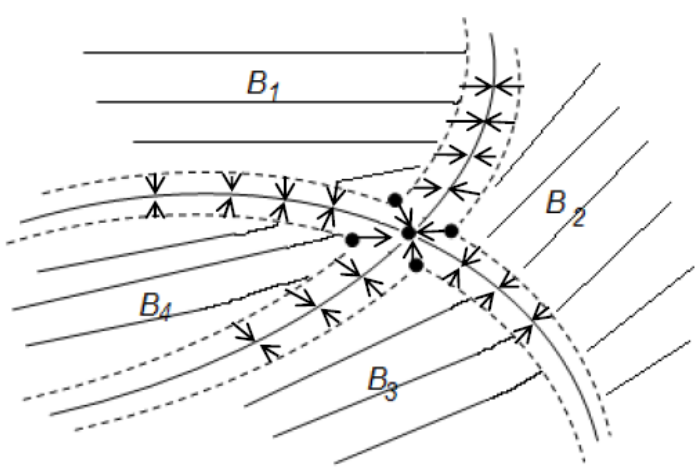

Figure 10: Neighborhood of a vertex with valency 4 . 


\begin{tabular}{|c|c|c|c|c|}
\hline $\boldsymbol{q}_{\mathbf{1}}$ & $\boldsymbol{q}_{\mathbf{2}}$ & $\boldsymbol{q}_{\mathbf{3}}$ & $\boldsymbol{k}$ & $\boldsymbol{H}(\boldsymbol{f})$ \\
\hline 2 & 2 & $p, p>1$ & $4 p$ & $\mathrm{D}_{2 n}$ \\
\hline 2 & 3 & 3 & 24 & $\mathrm{O}$ \\
\hline 2 & 3 & 4 & 48 & $\bar{O}$ \\
\hline 2 & 3 & 5 & 120 & $\overline{\mathrm{T}}$ \\
\hline
\end{tabular}

Table 1: Possibilities list.

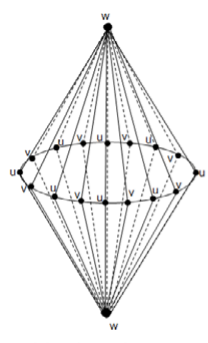

(a) $(2,2,8)$

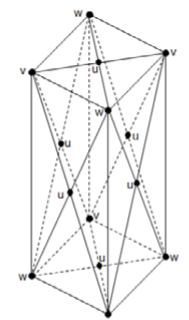

(b) $(2,3,3)$

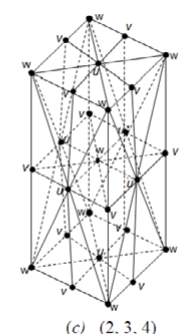

(c) $(2,3,4)$

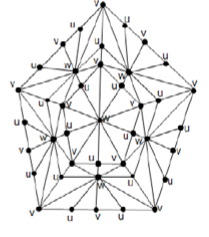

(d) $(2,3,5)$
Figure 11: Triangulation.

\section{Balanced folding over a polygon}

Equations (1) and (2) can be refined slightly when $f$ is balanced. In this case, if we label the vertices of the polygon $P n$ as $V_{1}, \ldots, V n$, then each vertex in the set $f^{-1}(V j)$ has the same valency $2 q_{j}, j=1, \ldots, n$. It follows that $f^{-1}(V j)$ contains $\frac{k}{2 q_{j}}$ elements. Thus the number of
vertices of $\Gamma_{f}$ is:

$$
V=\frac{k}{2} \sum_{j=1}^{n} \frac{1}{q_{j}}
$$

Hence for a balanced folding over a disc, equation (3) may be reduced to

$$
2 e(M)=k(2-n)+k \sum_{j=1}^{n} \frac{1}{q_{j}}=k\left\{(2-n)+\sum_{j=1}^{n} \frac{1}{q_{j}}\right\}
$$

Certain cases of equation (4) are of special interest. For instance, let $n=3$, so that $M$ is triangulated by $\Gamma_{\rho}$ and equation (4) becomes

$$
2 e(M)=k\left\{\frac{1}{q_{1}}+\frac{1}{q_{2}}+\frac{1}{q_{3}}-1\right\}_{1}
$$

Thus if $M$ is a sphere, then $\frac{1}{q_{1}}+\frac{1}{q_{2}}+\frac{1}{q_{3}}>1$ and $k \geq 4$. The only possibilities are listed in the following Table 1:

The group $H(f)$ associated with $f$ according to Theorem 1 is shown in column5, and the corresponding triangulation of $S^{2}$ are shown in Figures 11a-11d. Note that in Figure 11d we have drawn only one side. The vertices are labeled in such a way that vertices with the same image under $f$ are labelled alike.

\section{References}

1. Kinsey LC (1993) Topology of surfaces. Springer, New York, USA.

2. El-Kholy E, Shahin RM (1998) Cellular folding. J Inst Math and Comp Sci 11 177-181.

3. Robertson SA, El-Kholy E (1986) Topological foldings. Commun Fac Sci Univ Ank Ser 35: 101-107.

4. Farran HR, El-Kholy E, Robertson SA (1996) Folding a surface to a polygon Geometriae Dedicata 63: 255-266. 